\title{
PLASTIC LIMIT LOAD OF ELLIPSOIDAL PRESSURE VESSEL HEAD WITH NOZZLE UNDER INTERNAL PRESSURE LOADING
}

\author{
V.N. SKOPINSKY*, N.A. BERKOV and A.B. SMETANKIN \\ Moscow State Industrial University \\ Avtozavodskaya 16, 115280, Moscow, RUSSIA \\ e-mail: skopin-j@mail.msiu.ru
}

\begin{abstract}
A new method and numerical procedure for determining the plastic limit load in an ellipsoid-cylinder intersection using the elastic-plastic finite element analysis are presented. The proposed method is based on the maximum criterion of the rate of change of the relative plastic work. For the elastic-plastic analysis of the nozzle connections the 2D finite element method and plasticity theory with strain hardening are used. The results of the comparison of the plastic limit pressure obtained on the basis of different known criteria and the proposed criterion are presented. A parametric study of ellipsoidal heads with a nozzle under internal pressure loading was performed. The effects of nondimensional geometric parameters of shell intersection on the plastic limit pressure are discussed.
\end{abstract}

Key words: shell intersection, elastic-plastic finite element analysis, plastic limit load, ellipsoidal head, nozzle connection.

\section{Introduction}

A shell structure in the form of ellipsoidal head with a cylindrical nozzle belongs to one of the typical configurations in many industries, such as petrochemical and chemical machine-building, power engineering, etc. Such configurations are considered usually as intersecting shells. The local stress concentration in the intersection region caused by a geometric discontinuity can be very meaningful. An analysis of the intersecting shells forms an important class of applied problems in mechanics of shells and the methods of analysis of intersecting shells are important in scientific and engineering studies.

In the engineering practice, in some cases, a linear analysis is sufficient. However, a nonlinear analysis is a more accurate and appropriate research solution for the definition of the specified allowable loads in pressure vessels and has been adapted by the well-known Pressure Vessel Design Codes (ASME 2004; PD 5500 2006; EN 13445 2002).

The elastic analysis of ellipsoidal heads with nozzles and a parametric study have been considered by Skopinsky et al. $(1994 ; 2000 ; 2003 ; 2006)$ and in the book of Skopinsky $(2008)$. Hsieh et al. $(2000 ; 2002)$ described the elastic and inelastic analyses and definition of plastic load in torispherical heads with large nozzles. Experimental data and results of 3D FEA for individual loads and combined loading were presented there as well.

The objective of this paper is to describe the method for determining the plastic limit load in ellipsoidal heads with cylindrical nozzles and to study the influence of various geometric parameters on the plastic limit load.

\section{Determining plastic limit load}

Determining the plastic limit load producing gross plastic deformation is of great importance for nozzle connections. A review of several methods (criteria) for determining plastic loads is given by Gerdeen

\footnotetext{
* To whom correspondence should be addressed
} 
(1979). In practice, two methods are most commonly used in the analysis of pressure vessels with nozzles: the Twice Elastic Slope (TES) method and the Tangent Intersection (TI) method. Both methods are based on a characteristic load-deformation curve and are empirical procedures.

Muscat et al. (2003) proposed a work criterion for evaluating plastic limit loads (Plastic Work or PW criterion). This method is based on the plastic work dissipated in a structure from a ductile material as loading progresses. A characteristic curve is drawn as load (or a load parameter for combined loads) versus plastic work dissipation in the structure. The plastic limit load by the tangent intersection method is defined as the intersection between a straight-line tangent from the curve and the load vertical axis.

Gerdeen (1979) noted that the TES and TI methods have practical difficulties in selecting the appropriate load parameter for combined loading. The TI and PW criteria have one common drawback: it is not always clear precisely, where the tangent to the characteristic curve should be drawn, especially if the curve does not display a plateau. Therefore, the subjective choice of the point on the curve for the tangent can affect the defined plastic limit load.

Li and Mackenzie $(2005 ; 2006)$ proposed a new criterion based on the plastic work concept as well. The plastic limit load is defined by relating the curvature of the load-plastic work curve to specific stages in the evolution of the gross plastic deformation mechanism (Plastic Work Curvature or PWC criterion). The results of the example presented for the branch intersection assuming a strain hardening material have shown that the PWC criterion gives a higher value of plastic load than other criteria considered (TES, TI and PW criteria). The reason may be an ambiguous condition of this criterion.

Camilleri et al. (2006) developed the RPWC (Ratio Plastic Work curvature) criterion. The development of the gross plastic deformation mechanism is characterized by plotting the ratio of plastic work to total work against the applied load. Plastic load is identified at the load equivalent to the maximum curvature. However, the application of RPWC criterion to the analysis of a head with a nozzle was not considered.

Some comments on the deformation parameters which are used for the characteristic curve can be made. Conventionally, deformation parameters can be divided into local and global. These parameters should adequately represent the development of ductile material yielding in an elementary volume or a global process of the elastic-plastic deformation in a structure as a whole.

A local deformation parameter is selected for a point (elementary volume of the body); for example, it can be a strain component, a strain intensity, the largest principal strain or a displacement component at a point in the plastic region. The advantage of using a local deformation parameter is the tracking of its changes, usually in the area of the most significant plastic deformation. The disadvantage is that this change may be alternating, which leads to a non-monotonic variation of the load-deformation curve or a strongly nonlinear load-deformation dependence which complicates the correct definition of limit load. In this regard, in our opinion, in an inelastic analysis it is preferable to use the strain intensity, the plastic strain intensity or the specific plastic work as the local deformation parameter, as they are always positive and increasing quantities. Also, as noted by Gerdeen (1979), the estimating load is dependent upon the location of the strain or deflection measurement.

The global deformation parameter may be the plastic work of the shell intersection or displacement at any characteristic point of a vessel or a nozzle located outside the region of plastic deformation. The advantage of the global deformation parameter is that it is always growing and the characteristic curve is monotonically increasing as a consequence.

\section{Ellipsoid-cylinder intersection. Finite element modeling}

For the elastic-plastic analysis of the nozzle connection in an ellipsoidal pressure vessel head, the 2D finite element method, a small deformation shell theory, a plasticity theory with strain hardening and the Von Mises yield criterion are used.

A nozzle connection is considered as an intersection of two shells: an ellipsoidal shell of revolution (a basic shell) and a cylindrical shell (a nozzle). A common case of the nozzle position in the ellipsoidal shell is shown in Fig.1: the geometry presented corresponds to intersection of middle shell surfaces. 


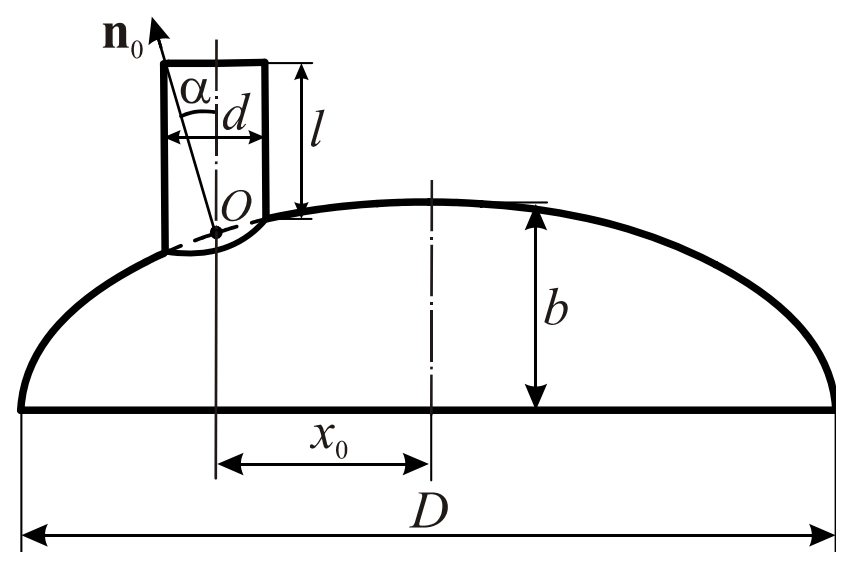

Fig.1. Geometry of ellipsoid-cylinder intersection.

For the ellipsoid-cylinder intersection, the main plane which passes through the axis of the basic shell and the normal $\boldsymbol{n}_{0}$ to the ellipsoid surface at the point of the intersection of this surface by the nozzle axis is introduced. The parameter $\alpha$ defines the angular deflection of the nozzle axis from the radial position (from the normal $\boldsymbol{n}_{0}$ ), the parameter $x_{0}$ defines the offset nozzle displacement from central position.

With the purpose of a parametric study, the typical nozzle connections in an ellipsoidal head are considered following Skopinsky (2008): radial $(\alpha=0)$, non-radial $(\alpha>0)$; central $\left(x_{0}=0\right)$ and non-central $\left(x_{0} \neq 0\right)$.

The finite element modelling of the shell intersection includes the following specific features: the application of curvilinear coordinate systems for individual shells, the use of a modified mixed variational formulation and 2D shell-curved element with a special scheme for displacement and strain approximations, and the use of a rational calculation algorithm with minimum of coordinate transformations. The shell element model used is an arbitrary doubly curved quadrilateral element with 20 degrees of freedom, five at each node (three displacements and two rotations in principal coordinate planes). In the numerical analysis, taking into account the nonlinear stress distribution in the elastic-plastic range, a layered element model is used. The shell element is divided into 10 layers over the thickness and the variation of material properties from layer to layer is accounted for. The element characteristics are determined by numerical integration over the element volume.

The elastic-plastic finite element analysis of the shell intersections is carried out using the initial stress technique proposed by Nayak and Zienkiewicz (1973) which allows one to perform calculations for any stress-strain dependence including an elastic-perfectly plastic material model or a stress-strain curve with a «yield plateau». For the elastic-plastic analysis of the intersecting shells in such a formulation, a developed specialized computer program SAIS is applied.

\section{Maximum criterion of rate of change of relative plastic work}

The numerical elastic-plastic analysis of the intersecting shells showed that for determining the plastic limit load, in our view, it is preferable to use a global deformation parameter - plastic work. The following reasons favorably distinguish this parameter from the others: (a) the parameter is an objective and revealing indicator of the mechanism of plastic deformation in the structure and the gradual change of the structure behavior in the process of loading, (b) as a result of the aforesaid, the characteristic curve is a smooth and monotonically increasing curve, and (c) the parameter can be used for structures subject to a single load or combined loading. However, the known criteria based on the plastic work do not always contain clear enough conditions to determine the plastic limit load.

Here, a new criterion based on the plastic work concept is proposed to substantiate a procedure for 
the definition of the plastic limit load. For this, consider some general magnitudes characterizing the elasticplastic deformation of a structure.

In the SAIS program using the FEA for the shell intersection configuration and following Iliushin (1948), the specific (per unit volume) plastic work $\bar{W}_{p}$ and the specific total work of deformation $\bar{W}$, the total plastic work $W_{p}$ and the total deformation work $W$ are defined as follows:

$$
\begin{aligned}
& \bar{W}_{p}=\int \sigma_{i} d \varepsilon_{i}^{p}, \\
& W_{p}=\sum_{N_{p e}} W_{p}^{e}=\sum_{N_{p e} V^{e}} \int_{W_{p}} d V, \\
& \bar{W}=\int \sigma_{i} d \varepsilon_{i}+0,5 K \theta, \quad W=\sum_{N_{e}} W^{e}=\sum_{N_{e}} \int V^{e} \bar{W} d V
\end{aligned}
$$

where $\sigma_{i}$ is the stress intensity (or equivalent stress), $\varepsilon_{i}^{p}$ is the plastic strain intensity (or equivalent plastic strain), $\varepsilon_{i}$ is the strain intensity, $K$ is the volumetric elastic modulus, $\theta$ is the volumetric strain, $N_{p e}$ is the number of elements in which the yielding takes place, and $N_{e}$ is the total number of elements.

For the analysis of the elastic-plastic deformation of the shell intersection the following parameters were introduced:

- relative plastic work

$$
C_{p}=\frac{W_{p}}{W}
$$

- the rate of change of relative plastic work with increasing load (or load parameter) $q$

$$
C_{p}^{\prime}=d C_{p} / d q
$$

The parameter $C_{p}\left(0 \leq C_{p}<1\right)$ is the global indicator of the plastic deformation of intersecting shells, where the function $C_{p}(q)$ is always monotonously increasing.

The results obtained using elastic-plastic analyses for different shell intersections show that the function $C_{p}^{\prime}(q)$ does not change monotonically and has a local maximum at a certain load. The presence of this maximum can be explained as follows. The features of the local stress distributions in intersecting shells has been discussed in Skopinsky (2008). As a consequence of this features, the first plastic strain appears in a relatively small area where the largest stresses exit. Then, as the load increases, the plastic strain spreads through the thickness of shells (nozzle and vessel or branch and main run), along the intersection region and away from it. Initially, the value of the plastic work increases intensively $\left(C_{p}^{\prime}>0\right)$ due to the active stress redistribution in the region of plastic deformation. Then, the plastic work gradually becomes the dominant part of the total deformation energy of the structure. The maximum value $C_{p}^{\prime}$ characterizes the establishment of such a plastic deformation mechanism for a shell intersection when, as calculations have shown, with further increasing load, the rate of increase of the total work $W$ is defined by the rate of increase in the plastic work $W_{p}$. Thereafter, the rate of change of the relative plastic work is reduced.

As a result, the maximum criterion of the rate of change of the relative plastic work is proposed to determine the plastic limit load $\left(q_{p l}\right)$. 
In the SAIS program, the definition of the plastic limit load $q_{p l}$ is as follows. For a «load program» (given load values $q_{j}, j=1, \ldots, n$ ), the values of parameter $C_{p, j}$ from the numerical results of the FEA are calculated for each load step according to Eqs.(4.1) - (4.4). These results are written into a data file as a series of $q-C_{p}$ points. A cubic spline approximation of the function $C_{p}(q)$ through $q-C_{p}$ data points is generated to find the derivative $C_{p, j}^{\prime}$ at each point using a standard procedure. Then, for the obtained function $C_{p}^{\prime}(q)$, the plastic limit load $q_{p l}$ is defined for the point of this function where the derivative reaches its maximum (graphically or using numerical iterative procedure).

The application of this method for the definition of the plastic limit pressure in the intersecting cylindrical shells was presented by Skopinsky and Berkov (in press). In addition, in this paper the criterion of specific plastic work of a characteristic curve $q-\bar{W}_{p \text {, max }}$ was proposed for the definition of the plastic limit load. In our opinion, the specific plastic work is a reasonable local deformation parameter, and the application of two criteria on the basis of global and local deformation parameters gives upper and lower estimations of plastic limit load.

\section{Example analysis}

In the pressure vessel industry, the internal pressure is the primary load. Usually, the standard ellipsoidal heads 2:1 are used in practical design. For example, the application of the proposed criterion for determining the plastic limit pressure is demonstrated for the ellipsoidal head with radial central nozzle $(\alpha=$ $0, x_{0}=0$ ) under internal pressure $p$. The nozzle connection has the following dimensions (see Fig.1): mean diameter of the ellipsoidal head $D=600 \mathrm{~mm}$, head height $b=150 \mathrm{~mm}$, mean diameter of the nozzle $d=60$ $\mathrm{mm}$, nozzle length $l=180 \mathrm{~mm}$. The head and nozzle are of equal thickness $H=h=6 \mathrm{~mm}$.

It is assumed that the material for the shells is steel 20nc (low carbon steel), for which the mechanical properties and engineering stress-strain curve were obtained during testing standard specimens at the MSIU Laboratory of Material Strength: Young's modulus $E=210 \mathrm{GPa}$, yield strength $\sigma_{y}=309 \mathrm{MPa}$ and ultimate tensile strength $\sigma_{u}=465 \mathrm{MPa}$. A multilinear elastic-plastic material model was employed using eleven points from the true stress-strain relationships of the material which are given in Tab.1.

Table 1. Multilinear material model.

\begin{tabular}{|c|c|c|c|c|c|c|c|c|c|c|c|}
\hline Point & 1 & 2 & 3 & 4 & 5 & 6 & 7 & 8 & 9 & 10 & 11 \\
\hline Stress $(M P a)$ & 309 & 313 & 318 & 324 & 397 & 437 & 467 & 506 & 531 & 544 & 553 \\
\hline Strain $(\%)$ & 0.148 & 1.76 & 1.86 & 2.06 & 3.94 & 5.75 & 7.51 & 10.82 & 13.92 & 15.45 & 16.76 \\
\hline
\end{tabular}

Figure 2a shows a plot of the relative plastic work $\left(C_{p}\right)$ versus load $p$ using a data file obtained for a load series. The corresponding curve indicating the variation of the rate of the change of the relative plastic work $\left(C_{p}^{\prime}\right)$ for an increasing load and the definition of the plastic limit pressure $\left(p_{p l}\right)$ is shown in Fig.2b. As it can be seen, the $C_{p}^{\prime}-p$ curve has a pronounced maximum, according to which the plastic limit load can be designated. 
a)

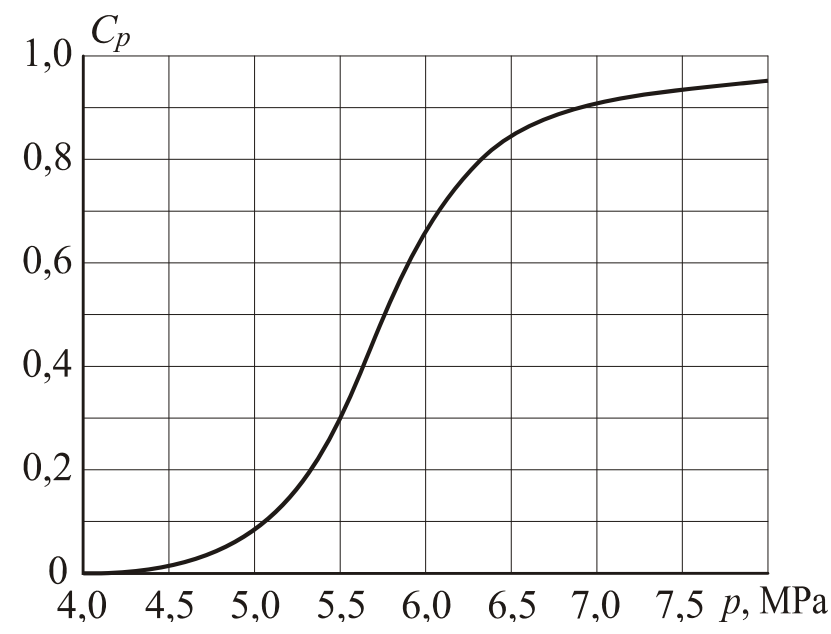

b)

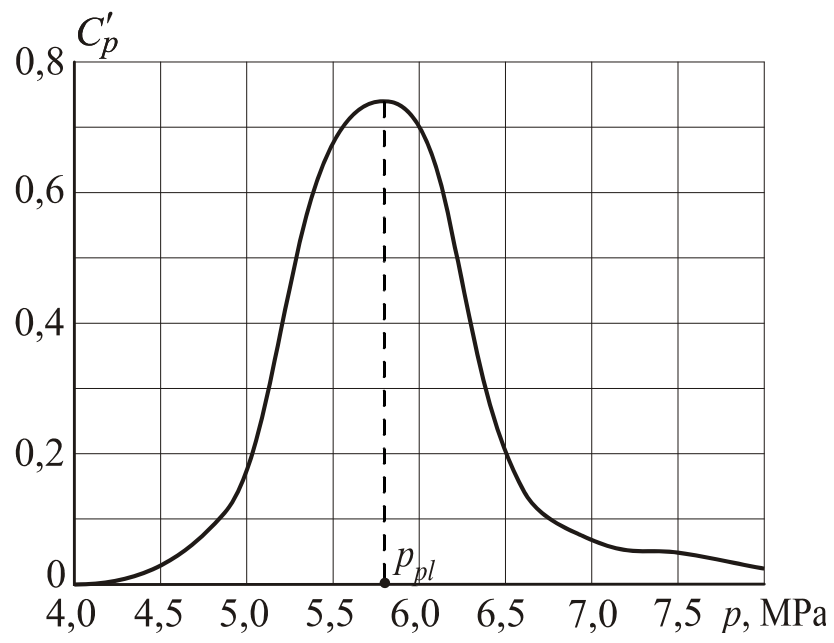

Fig.2. Maximum criterion of rate of change of relative plastic work: (a) plot of relative plastic work; (b) plot of rate of relative plastic work.

For comparison, the plastic limit pressure was obtained also on the basis of different criteria (TES, TI, PW) and the local deformation parameter $\bar{W}_{p, \max }$. The characteristic curves $\left(p-u_{n}\right),\left(p-\varepsilon_{i, \max }\right),\left(p-W_{p}\right)$ and $\left(p-\bar{W}_{p, \max }\right)$ and the application of different methods for the definition of the plastic limit pressure are shown in Fig.3. 


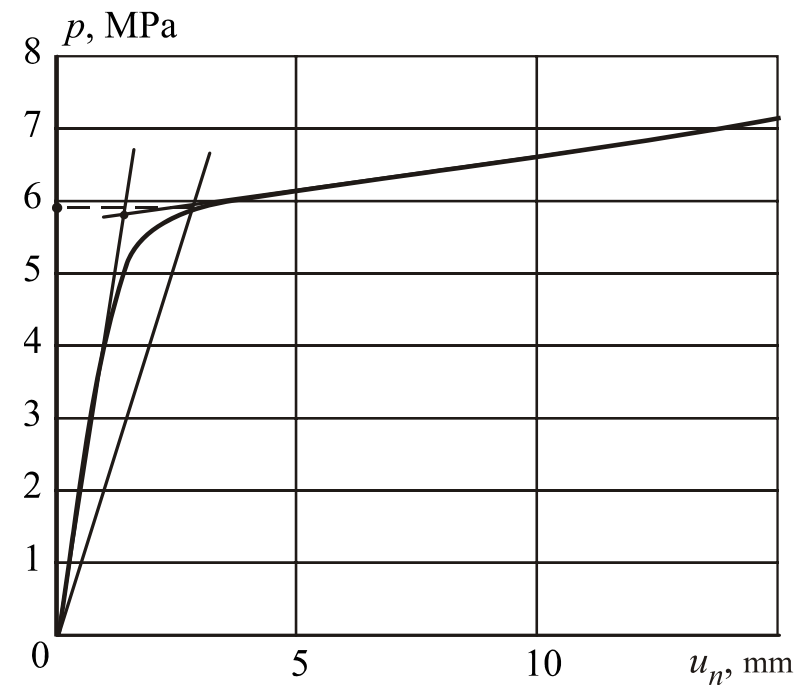

(a)

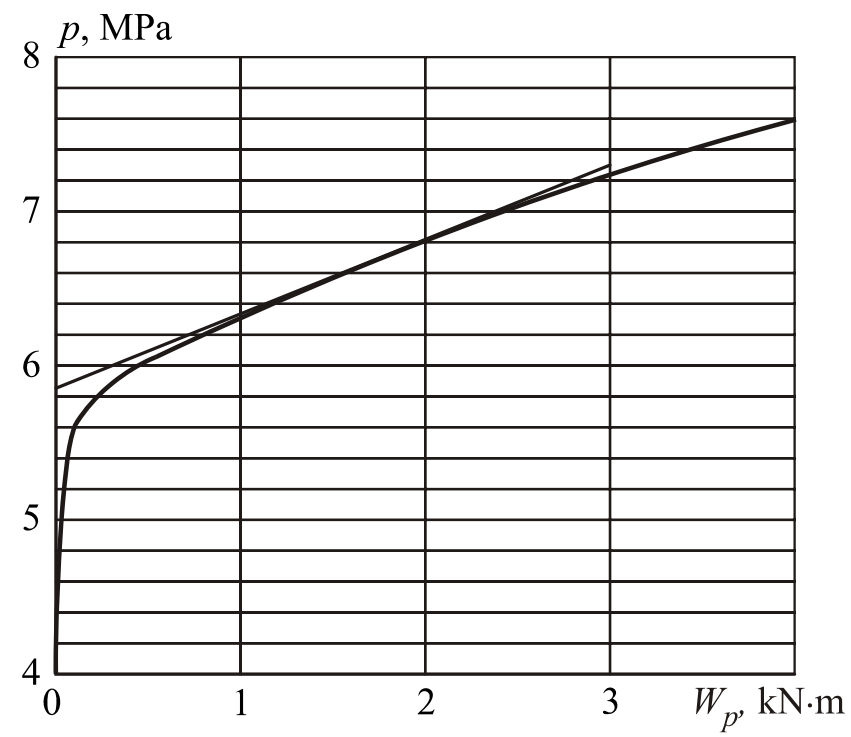

(c)

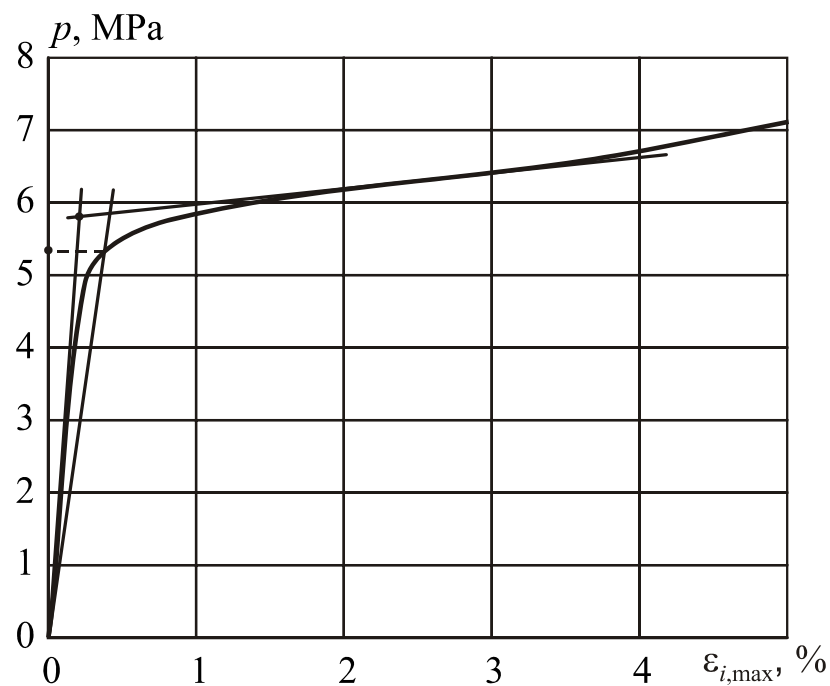

(b)

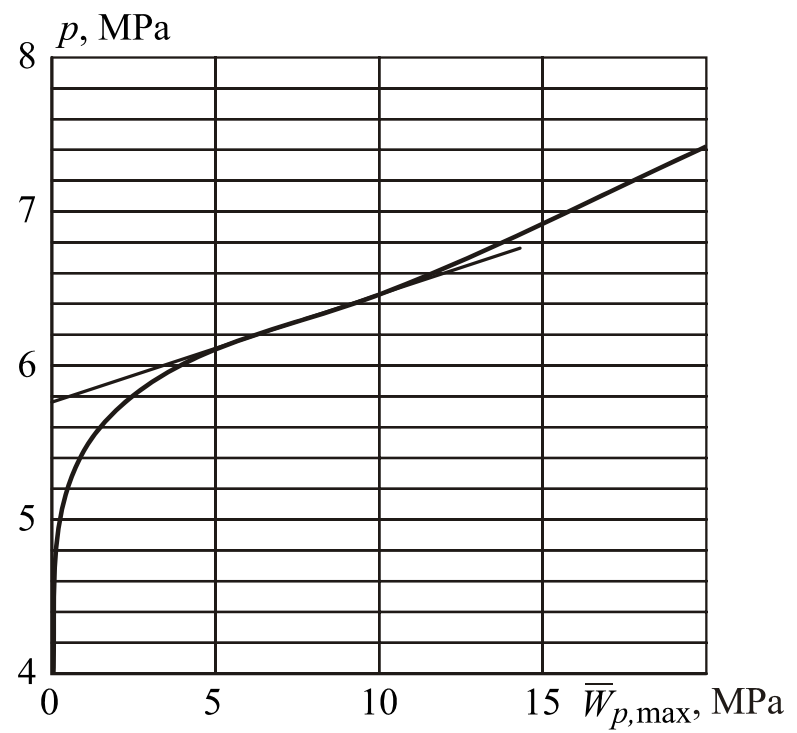

(d)

Fig.3. Characteristic curves for ellipsoidal head with central radial nozzle: (a) pressure - nozzle end axial displacement; (b) pressure - maximum strain intensity; (c) pressure - plastic work; (d) pressure maximum specific plastic work.

Here $u_{n}$ represents the nozzle end axial displacement and $\varepsilon_{i \text {,max }}$ the maximum strain intensity in shell intersection. In all cases, the tangent to the plastic part of the characteristic curve was drawn through a point corresponding to the maximum plastic strain intensity equal to $3 \%$.

A comparison of the results for the plastic limit pressure obtained on the basis of different criteria is given in Tab.2. 
Table 2. Comparison of plastic limit pressures using different criteria.

\begin{tabular}{|c|c|c|c|c|c|c|c|}
\hline Curve & \multicolumn{2}{|c|}{$p-u_{n}$} & \multicolumn{2}{c|}{$p-\varepsilon_{i, \max }$} & $p-W_{p}$ & $p-\bar{W}_{p, \max }$ & $C_{p}^{\prime}-p$ \\
\hline Method & TES & TI & TES & TI & TI & TI & $\max C_{p}^{\prime}$ \\
\hline$p_{p l}, M P a$ & 5.90 & 5.80 & 5.34 & 5.77 & 5.85 & 5.76 & 5.81 \\
\hline
\end{tabular}

As it might be expected, the use of the local deformation parameter $\varepsilon_{i \text {,max }}$ gives the lowest values of the plastic pressure for TES criterion because the characteristic curve reflects the effects of the plastic deformation in elementary volume of the material, which increase most intensively. At the same time, the application of the TI method for all curves gives very close values. The plastic pressure obtained on the basis of the proposed criterion is in good agreement with other results.

Thus, the results presented demonstrate that the proposed method on based on the maximum criterion of the rate of change of the relative plastic work is a convenient tool for the determination of the plastic limit pressure. It can also be noted that the results demonstrate some useful application of the two proposed criteria based on the global and local deformation parameters to estimate the plastic limit pressure. In this case, we can obtain both upper and lower estimations for the plastic limit load.

\section{Parametric study}

For the ellipsoid-cylinder intersections, important nondimensional geometric parameters having influence on the stress state in shells and the plastic limit load are the following

$$
\frac{d}{D}, \quad \frac{D}{H}, \quad \frac{h}{H}, \quad \bar{b}=\frac{b}{a}, \quad \alpha, \quad x_{0}^{\prime}=\frac{x_{0}}{a} .
$$

Parameters $d / D$ and $h / H$ are characteristics of the intersection in general. The diameter ratio $d / D$ defines the extent of the weakening of the head by the intersection hole. The thickness ratio $h / H$ defines the relative stiffness of the intersecting shells and significantly affects the stress concentration in the shells. The parameter $D / H$ (as well as $d / h$ for the nozzle) is characteristic of an individual shell.

In the elastic-plastic analysis, except for the parameters (6), mechanical properties of the material including its integral characteristic such as the stress-strain curve influence the plastic limit load.

Using the SAIS program, the parametric elastic-plastic study for model nozzle connections in an ellipsoidal head under pressure loading was performed. The influence of the geometric parameters (6) on the plastic limit pressure was studied.

Due to symmetry, only half of the shell intersection was used for analysis. Symmetric boundary conditions were imposed on the symmetry plane; the boundary conditions at the edge section of the nozzle corresponded to the flange fixing (axial forces from the internal pressure were applied to the nozzle), and edge section of the ellipsoidal head corresponded to a simply supported shell (meridional and circumferential displacements, and two rotations are zero). As an example, Fig.4 illustrates the finite element model of ellipsoidal head with a non-central vertical nozzle. 


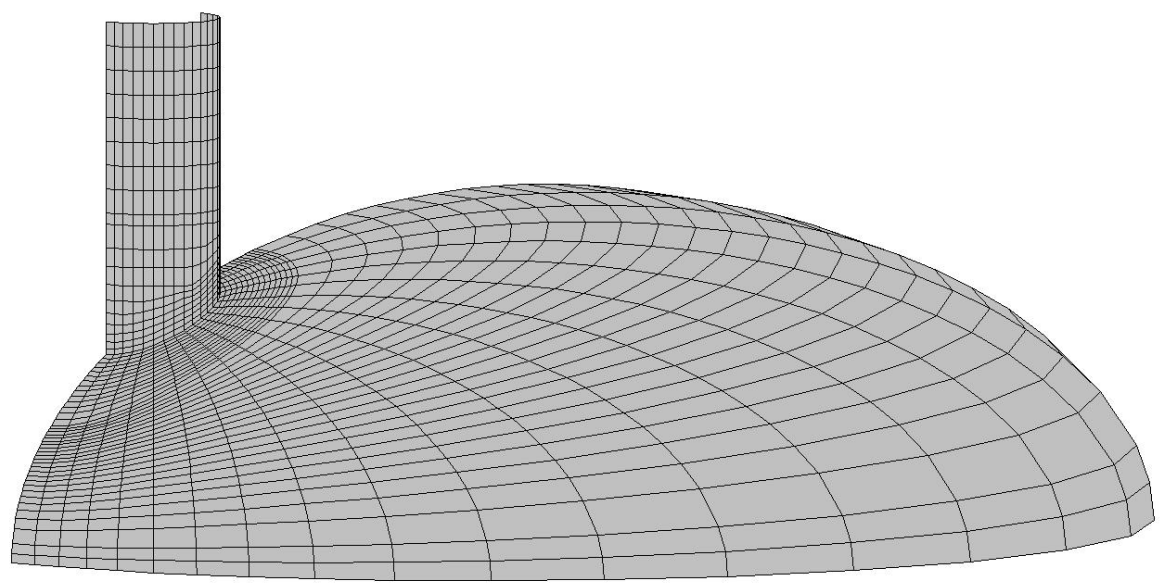

Fig.4. FE model of ellipsoidal head with a non-central vertical nozzle.

From the stress analysis conducted, some comments concerning the stress state in the intersecting shells under consideration subjected to internal pressure can be made. An ellipsoidal shell is a shell of double curvature and principal radii of the curvature change in the meridional direction. As a result, even membrane stresses change considerably in the meridional direction: thus, the circumferential stress from the pole to the edge of an ellipsoidal shell changes from tensile to compressive. The shell junction is a region of a high stress concentration caused by geometric discontinuity. Therefore, stresses in the vicinity of the intersection region change very intensively. In addition, the nozzle displacement from the central position and the angular parameter $\alpha$ has an influence on the stress distribution in shells.

In this work, the results of the parametric study are presented for the shell intersections with the following non-dimensional geometric parameters

$$
\bar{b}=0.5, \quad D / H=100, \quad l / d=3 .
$$

It is assumed that the material for the shells is steel $20 n c$, for which the mechanical properties are mentioned above. In the finite element modelling, a weld between a head and a nozzle was ignored.

The plastic limit pressure was calculated by applying the proposed maximum criterion of the rate of change of the relative plastic work. For the purpose of the parametric study, the normalized plastic limit pressure is defined as follows

$$
\bar{p}_{p l}=\frac{p_{p l}}{p_{y}}, \quad p_{y}=\frac{\sigma_{y}}{D / 2 H}
$$

Here $p_{y}$ represents the pressure at which the maximum stress intensity in the head (a ellipsoidal shell without a hole and $\bar{b}=0.5)$ is equal to the yield strength $\left(\sigma_{i}=\sigma_{y}\right)$.

The influence of the parameter $d / D$ is shown in Fig. 5 for the ellipsoidal head with the radial central nozzle $\left(\alpha=0, x_{0}=0\right)$.

The shell intersections with different thickness ratio are considered $(h / H=1.0$ and $h / H=0.6)$. Results presented demonstrate an evident trend to reduce the value of the limit pressure by increasing the hole of the basic shell. For connections with $h / H<1.0$, in comparison with intersecting shells of equal thickness, there are an intensification of the bending effect in the shell of smaller thickness (nozzle) and an increase of the maximum stress for the shell intersection in general. As a result, the plastic limit pressure is reduced. 


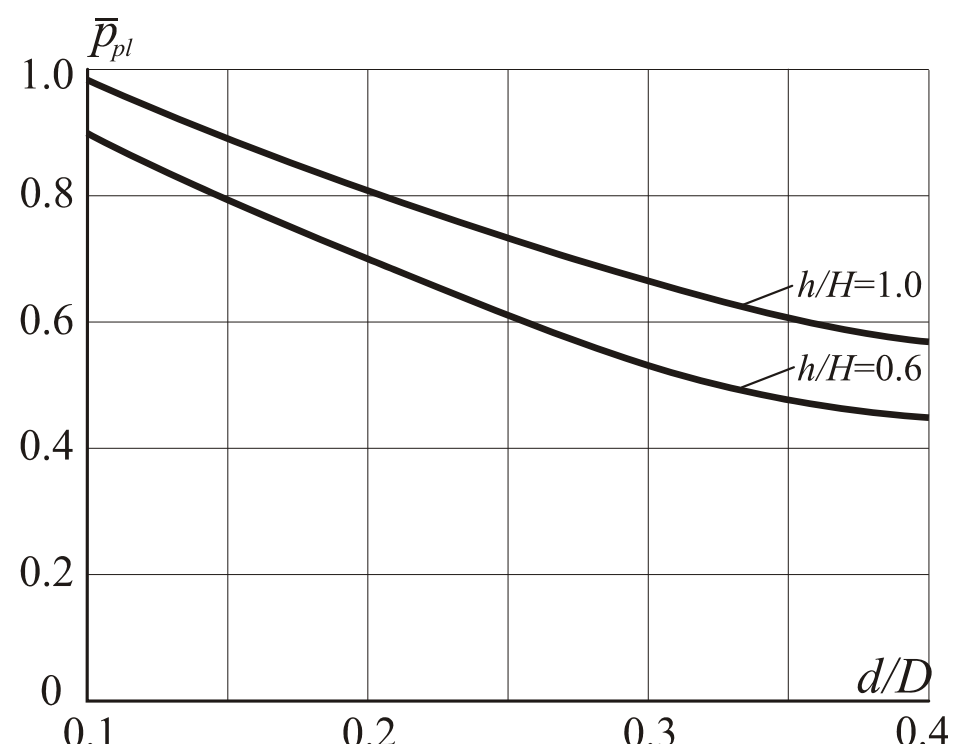

Fig.5. Effect of diameter ratio on plastic limit pressure.

The elastic-plastic analysis of the ellipsoidal heads with a non-radial nozzle indicated that the influence of the angular parameter $\alpha$ on the plastic limit pressure is not significant.

The investigation of the influence of the parameter of the offset nozzle displacement from the central position was performed for heads with vertical nozzle which are widely used in pressure vessels. The value of the angle $\alpha_{v}$ defining the vertical position of the nozzle axis is given by the following expression

$$
\alpha_{v}=\operatorname{arctg}\left(\frac{\bar{x}_{0} \cdot \bar{b}}{\sqrt{1-\bar{x}_{0}^{2}}}\right) .
$$

Figure 6 shows the effect of the parameter $\bar{x}_{0}$ on the plastic limit pressure for the shell intersections with $d / D=0.1$.

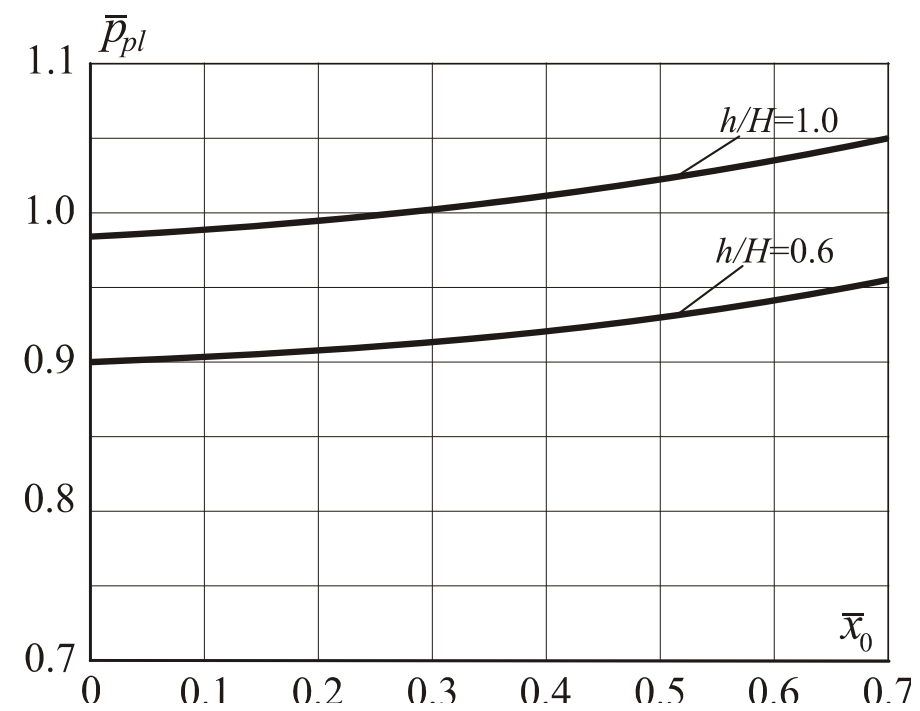

Fig.6. Effect of nozzle displacement from central position on plastic limit pressure. 
The results presented indicate a relatively small increase of the limit pressure when the nozzle is displaced from a central position. This effect can be explained by increasing the irregularity of the stress distribution in the intersection region and nearby, by the changing nature of stress components: the bending stresses become prevalent components which have less influence on the development of the plastic deformation in shells than membrane stress components. In addition, it can be noted that as the parameter $x_{0}$ increases, the intersection region is displaced in a stiffer part of the ellipsoidal shell.

\section{Conclusions}

The present paper describes the application of a new criterion for the definition of the plastic limit load using the two-dimensional elastic-plastic finite element analysis. The method proposed in contrast to other methods is not an empirical procedure and it can be realized both graphically, and in a computer program.

In this paper, results of the elastic-plastic stress analysis and determination of the plastic limit pressure for ellipsoidal heads with cylindrical nozzles are presented. Some results obtained in the parametric study indicate the influence of various geometric parameters on the plastic limit pressure and can be useful in pressure vessel design. In future, it is proposed to extend the research scope of the nonlinear analysis of intersecting shells.

\section{Acknowledgement}

This study was supported by the Ministry of Education and Science of the Russian Federation, project No. 14.B37.21.1133.

\section{Nomenclature}

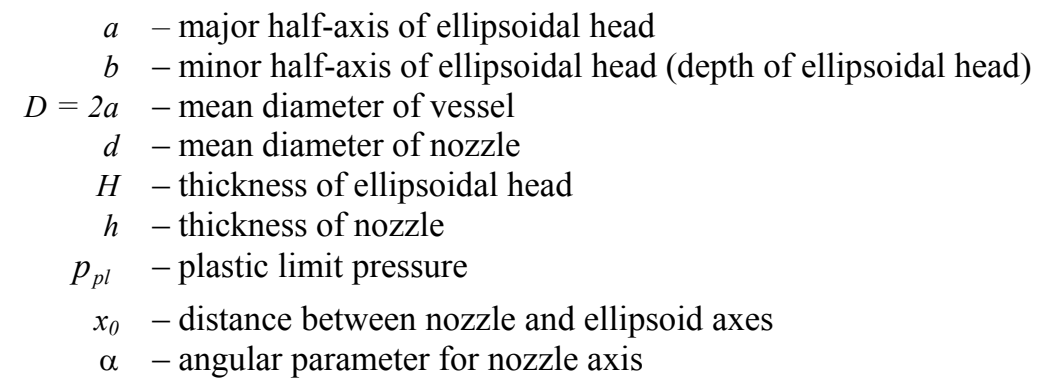

\section{References}

ASME (2004): Boiler and Pressure Vessel Code. Sections II and VIII. - New York.

BSI. PD 5500 (2006): Specification for Unfired Fusion Welded Pressure Vessels. -London: British Standards Institution.

Camilleri D., Mackenzie D. and Hamilton R. (2006): Evaluating plastic loads in torispherical heads using a new criterion of collapse. - ASME PVP Conference, Vancouver, Canada, vol.3, pp.701-708.

EN 13445 (2002): Unfired Pressure Vessels. Part 3: Design. European Committee for Standardisation (CEN), Brussels.

Gerdeen J.C. (1979): A critical evaluation of plastic behavior data and a unified definition of plastic loads for pressure components. - Welding Research Council Bulletin, No.254, pp.1-64.

Hsieh M.F., Moffat D.G. and Mistry J. (2000): Nozzles in the knuckle region of a torispherical head: limit load interaction under combined pressure and piping loads. - Int. J. Pressure Vessels and Piping, vol.77, No.13, pp.807-815. 
Hsieh M.F., Moreton D.N., Mistry J. and Moffat D.G. (2002): Limit loads for knuckle-encroaching nozzles in torispherical heads: experimental verification of finite element predictions. - J. Strain Analysis, vol.37, No.4, pp.313-326.

Iliushin A.A. (1948): Plastisity. - Moscow: GITTL.

Li H. and Mackenzie D. (2005): Characterising gross plastic deformation in design by analysis. - Int. J. Pressure Vessels and Piping, vol.82, No.10, pp.777-786.

Mackenzie D. and Li H. (2006): A plastic load criterion for inelastic design by analysis. - Trans. ASME, J. Pressure Vessel Technology, vol.128, No.1, pp.39-45.

Muscat M., Mackenzie D. and Hamilton R. (2003): A work criterion for plastic collapse. - Int. J. Pressure Vessels and Piping, vol.80, No.1, pp.49-58.

Nayak G.G. and Zienkiewicz O.C. (1973): Elasto-plastic stress analysis. A generalization for various constitutive relations including strain softening. - Int. J. Numer. Meth. Engn., vol.5, No.1, pp.113-135.

Skopinsky V.N. (2000): Stresses in ellipsoidal pressure vessel heads with noncentral nozzle. - Nuclear Engineering and Design, vol.198, pp.317-323.

Skopinsky V.N. (2008): Stresses in Intersecting Shells. - Moscow: Fizmatlit.

Skopinsky V.N. and Berkov N.A. (1994): Stress analysis of ellipsoidal shell with nozzle under internal pressure loading. - Trans. ASME, J. Pressure Vessel Technology, vol.116, No.3, pp.431-436.

Skopinsky V.N. and Berkov N.A. New criterion for the definition of plastic limit load in nozzle connections of pressure vessels. - Trans. ASME, J. Pressure Vessel Technology (in press).

Skopinsky V.N. and Smetankin A.B. (2003): Parametric study of reinforcement of pressure vessel head with offset nozzle. - Int. J. Pressure Vessels and Piping, vol.80, No.5, pp.333-343.

Skopinsky V.N. and Smetankin A.B. (2006): Modelling and stress analysis of nozzle connections in ellipsoidal heads of pressure vessels under external loading. - Int. J. Applied Mechanics and Engineering, vol.4, No.11, pp.965-979.

Received: May 22, 2013

Revised: July 18, 2013 